Raymund Pothmann

Zentrum Integrative Kinderschmerztherapie und Palliativmedizin, Hamburg, Deutschland

\title{
Palliativmedizin und Onkologie
}

Die Palliativversorgung hat in den letzten 15 Jahren einen gewaltigen Entwicklungsschub vollzogen und ist in der Gesellschaft angekommen. Ein breites Spektrum an Angeboten soll den unterschiedlichen Bedürfnissen gerecht werden. Als Standards hat sich die ärztliche und pflegerische Versorgung etabliert. Aber neben befriedigenden Pflegebedingungen und ärztlicher Behandlung besteht ein ausgeprägtes Bedürfnis nach komplementären Verfahren. Und hierbei gibt es gerade auf dem Traditionelle-chinesische-Medizin(TCM)-Sektor ein besonders breites Angebot, das die verschiedenen Wünsche gut abdecken kann. Und auch wenn die Beschwerden im Rahmen einer Krebserkrankung durch eine leitliniengerechte Palliativmedizin zu großen Teilen gelindert werden können, bleibt doch bei den Palliativpatienten unabhängig vom Krebs selbst ein großes Spektrum an TCM-Indikationen.

TCM-Angebote können komplementär und gleichzeitig im erweiterten Sinn als Spiritual Care begriffen werden. Gerade die somatopsychischen Aspekte der

Deutsche Zeitschrift für Akupunktur 2019. 62 (3): 179

https://doi.org/10.1007/s42212-019-0192-6

(c) Springer Medizin Verlag GmbH, ein Teil von

Springer Nature 2019
TCM bieten sich hierfür besonders an. Immer wieder erweist sich dabei der achtsame und respektvolle Umgang mit den betroffenen Patienten als tragende Basis.

\section{I) Der achtsame Umgang mit den betroffenen Patienten erweist sich als tragende Basis}

Im vorliegenden Heft wird darauf in einem breiten Spektrum von Zugangsverfahren eingegangen. Auch die Überbringung einer schlechten Nachricht wird dabei nicht ausgespart, steht sie doch ganz am Anfang einer palliativen Begleitung und fällt gerade kurativ tätigen Ärzten besonders schwer.

Auf dieser manchmal langen Gratwanderung zwischen „kurativ" und „palliativ“ bewähren sich komplementäre Möglichkeiten aus dem Bereich der TCM besonders und werden von den Patienten dankbar angenommen. TCM erweitert die Wahlmöglichkeiten für die Betroffenen, sodass sie mit den für sie passenden Verfahren behandelt werden können und allein dadurch wieder mehr "Lufthoheit“ bei der Bewältigung ihrer Erkrankung gewinnen können.

\section{Korrespondenzadresse}

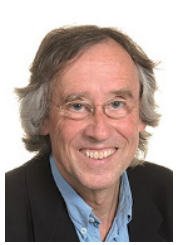

Dr. Raymund Pothmann Zentrum Integrative Kinderschmerztherapie und Palliativmedizin Alsterdorfer Markt 8, 22297 Hamburg, Deutschland pothmann@aol.com

Interessenkonflikt. R. Pothmann gibt an, dass kein Interessenkonflikt besteht. 